\title{
Prediction of Aero-Engine Exhaust Gas Temperature Based on Autoregressive Integrated Moving Average Model
}

\author{
Hong Zhang ${ }^{1,}$, Keqiang Dong ${ }^{2}$ \\ ${ }^{1}$ Basic Courses Department, Tianjin Sino-German University of Applied Sciences, Tianjin 300350, \\ China \\ ${ }^{2}$ School of Science, Civil Aviation University of China, Tianjin 300300, China \\ ahongzhangdong@163.com
}

Keywords: Aero-engine, Exhaust Gas Temperature, Autoregressive, Moving Average.

\begin{abstract}
Forecasting the performance of aero-engine is a crucial problem that facilitates the avoiding unnecessary delays of aircraft. By applying the Autoregressive Integrated Moving Average Model to aero-engine gas path system, we find that the ARIMA method is suitable for forecast Exhaust Gas Temperature fluctuation series. Further, we find the trend of predicted values and original values are similar and the error between them is small.
\end{abstract}

\section{Introduction}

Modern commercial aircraft require advanced prognostic and diagnostic schemes to determine engine performance in an effort to reduce operational and maintenance costs as well as limit aircrafts' downtime [1, 2]. For this purpose, Condition Based Maintenance (CBM), which decisions on operation and maintenance are based on the actual conditions reflected in sensor readings for aircraft are increasingly practiced by commercial airliners. Compared to traditional time based maintenance where maintenance schedules follow in regular intervals, CBM allows for the streamlining of maintenance procedures and potentially avoids unnecessary delays [3-5]. However, for the intention of streamlining maintenance procedures and avoiding unnecessary delays, the engine performance and health are need to estimate before upcoming flights.

This paper is focused on prediction of engine performance by applying Autoregressive Integrated Moving Average Model (ARIMA). The Autoregressive Integrated Moving Average Model offer the main advantage, ability to implicitly detect non-stationary in time series, catering for the complex non-stationary of aero-engine performance parameters.

The structure of the paper is as follows. In section 2, we focus on Autoregressive Integrated Moving Average Model enabling us to forecast the parameter characteristic of aero-engine. In section 3, we discuss the prediction of aero-engine Exhaust Gas Temperature time series. The conclusion of the paper is drawn in Section 4.

\section{Methodology and Algorithm}

\section{ARIMA methodology.}

ARIMA (p, d, q) methodology also allows models to be built that incorporate both autoregressive and moving average parameters together, where $\mathrm{p}$ represents the order of the autoregressive components, $\mathrm{d}$ is the number of differencing operators, and q means the highest order of the moving average term.

\section{The choice of (p,d,q).}

Here, we apply the ARIMA methodology to analyze the data of aircraft parameters collected at PW4000 aero-engine. Fig 1 show the Exhaust Gas Temperature (EGT) time series. By calculating the auto-correlative function and par-correlative function of the EGT series, we find that the EGT series is non-stationary and should be re-expressed such that they become stationary with respect to the variance and the mean. After first order difference of the original time series, the stationeriness is 
appeared (see Fig 2). And then, we employ the Minimum information criterion (AIC) to determine $\operatorname{ARIMA}(3,1,2)$.

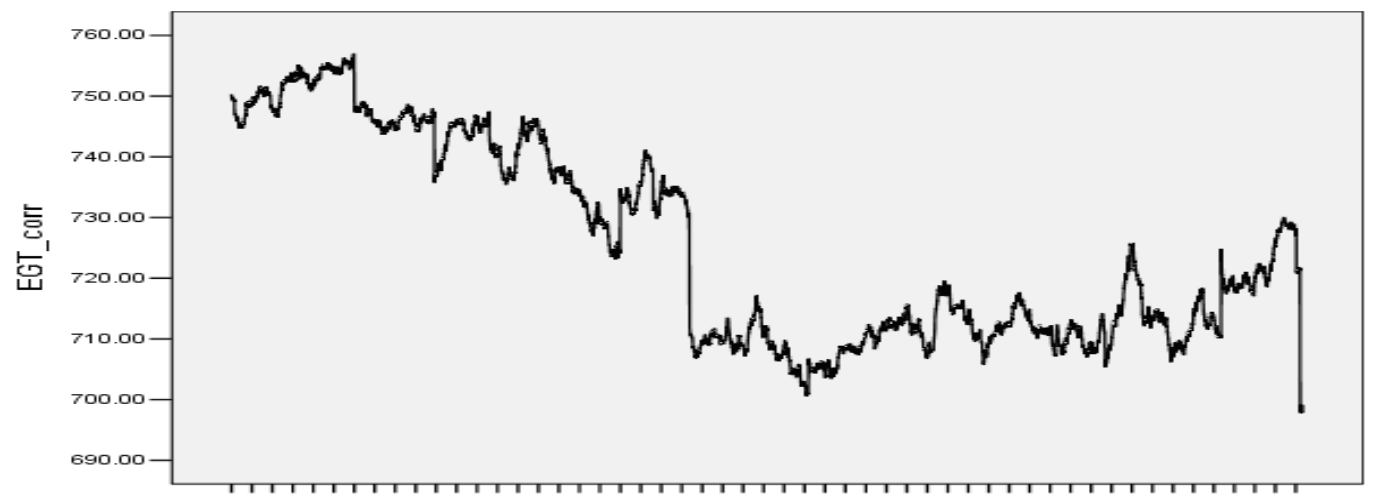

Fig. 1 the Exhaust Gas Temperature time series

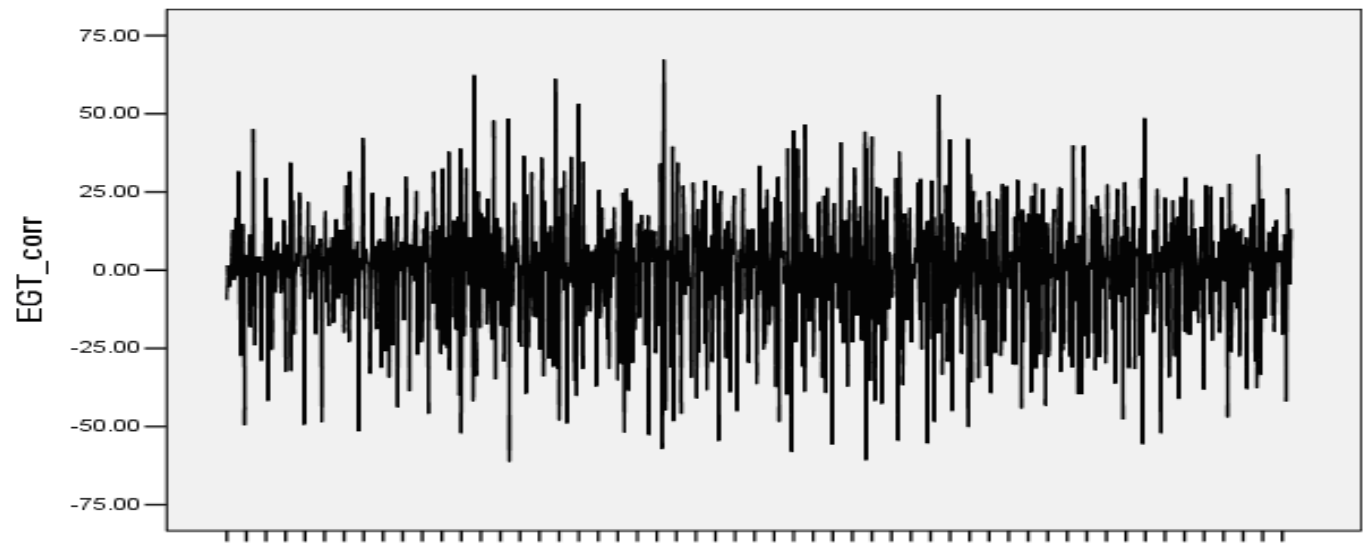

Fig. 2 the first order difference of Exhaust Gas Temperature time series

\section{ARIMA(3,1,2) Analysis.}

In order to test the advantages and disadvantages of the model, we test the residual sequence of the model. If the residual sequence is white noise, the model is appropriate for forecasting. By computing the $\mathrm{P}$-value of the residual sequence, we obtain the $\mathrm{p}=0.9999>0.05$ which indicate the residual sequence is white noise (see Fig 3).

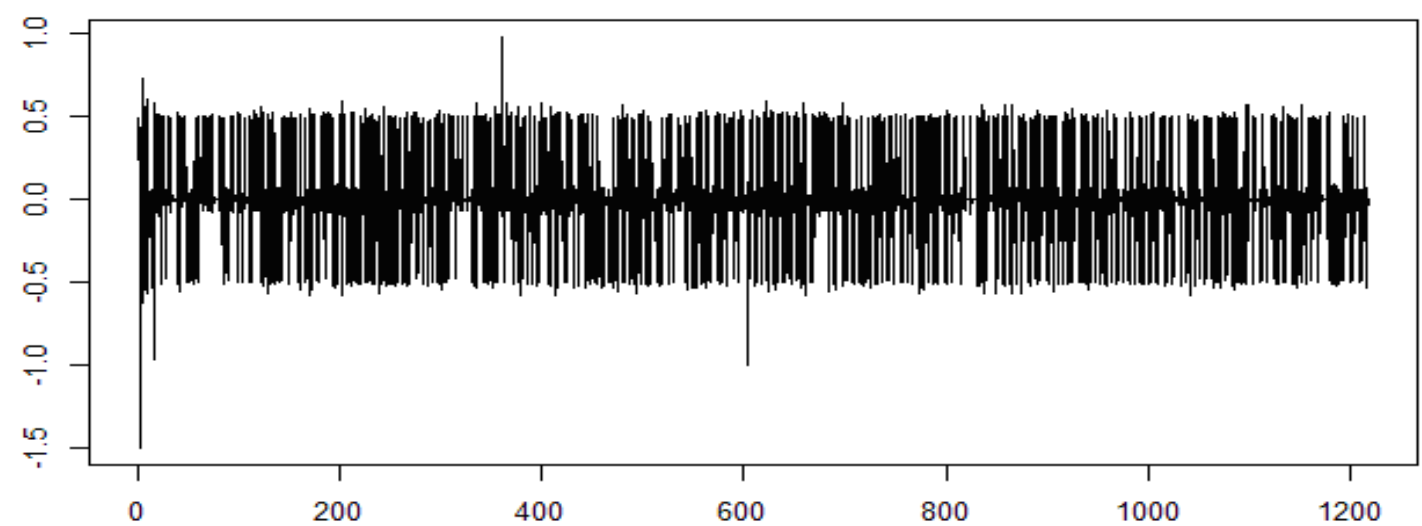

Fig. 3 the residual sequence of Exhaust Gas Temperature time series

\section{The residual sequence of Exhaust Gas Temperature time series}

For ARMA model, the maximum value and the minimum value of the prediction can be obtained by Eq.1 according to the desired confidence level. 


$$
\begin{aligned}
& U_{U}=\hat{W}_{K}(L)+T \bullet V(L) \bullet S_{e} \\
& U_{L}=\hat{W}_{K}(L)-T \bullet V(L) \bullet S_{e}
\end{aligned}
$$

where $S_{e}$ is the standard deviation of forecast error.

$$
\begin{aligned}
& S_{e}^{2}=\sum_{t=1}^{k} \hat{e}_{t}^{2} / k-p-q_{0} V(L) \\
& \varphi_{1}=\phi_{1}-\theta_{1} \\
& \varphi_{2}=\phi_{1} \varphi_{1}+\phi_{2}-\theta_{2} \\
& \ldots \\
& \varphi_{j}=\phi_{1} \varphi_{j-1}+\phi_{2} \varphi_{j-2}+\ldots+\phi_{p} \varphi_{j-p}-\theta_{j}
\end{aligned}
$$

Here, we perform a hypothesis test using 95\% confidence level, and obtain a p-value less than 0.05 . Therefore, ARIMA $(3,1,2)$ is suitable for forecast of EGT series. It can be seen from Fig. 4 that the trend of predicted values and original values are similar and the error between them is small.

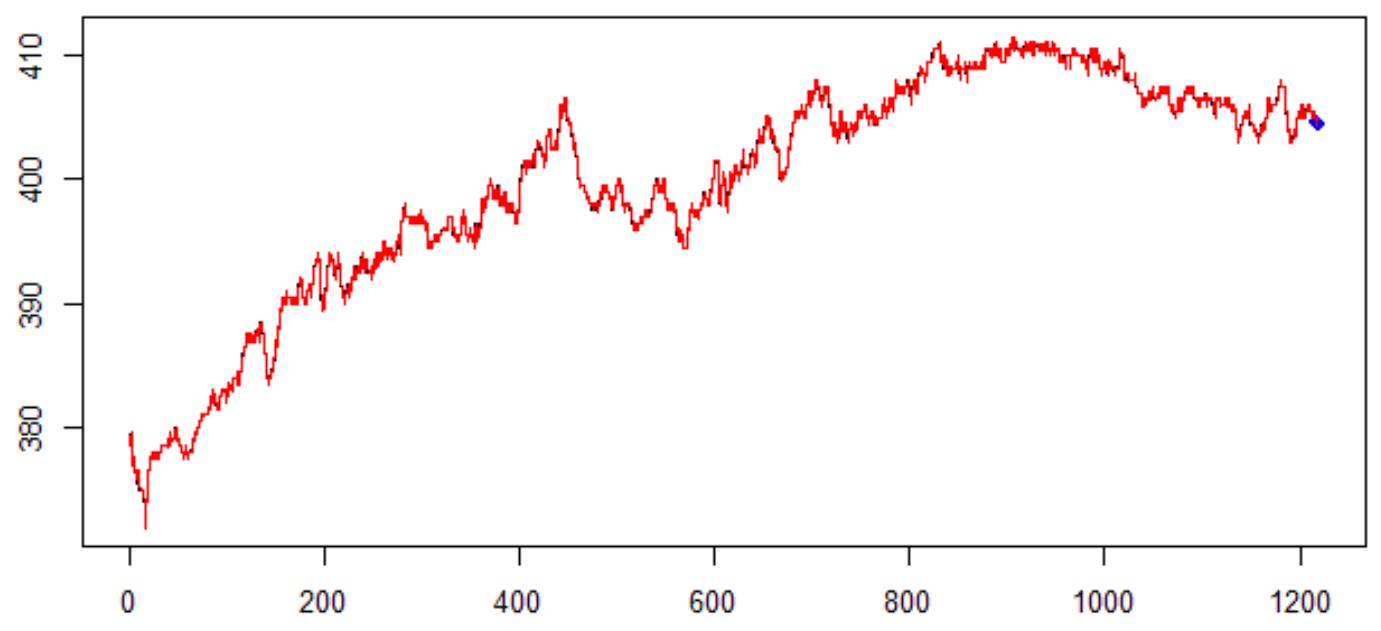

Fig. 4 the predictive values of Exhaust Gas Temperature time series

\section{Conclusions}

This paper introduced the Autoregressive Integrated Moving Average Model and applied it to aero-engine EGT time series. By employing the Minimum information criterion, we determine ARIMA $(3,1,2)$ model in this paper. And then, , we perform a hypothesis test using $95 \%$ confidence level, and obtain a p-value less than 0.05, which indicate the ARIMA method is suitable for forecast Exhaust Gas Temperature fluctuation series. Further, we calculate the error between the predicted values and original values and find the error is small.

\section{Acknowledgments}

The financial supports from the funds of the National Natural Science Foundation of China under Grant No. 61401467, the Fundamental Research Funds for the Tianjin Sino-German University of Applied Sciences under Grant No. zdkt2015-006, are gratefully acknowledged.

\section{References}

[1] A. Babbar, E. Ortiz, V. L. Syrmos, M. M. Arita, Advanced Diagnostics and Prognostics for Engine Health Monitoring, IEEE Aerospace Conference, Big Sky, U.S.A., 2009. 
[2] A. R. Yukitomo, V. L. Syrmos, Forecasting Gas Turbine Exhaust Gas Temperatures Using Support Vector Machine Experts and Genetic Algorithm, 18th Mediterranean Conference on Control \& Automation, Marrakech, Morocco, 2010.

[3] E. Ortiz, A. Babbar, V. L. Syrmos, Extreme Value Theory for Engine Health Monitoring and Diagnosis, 18th IEEE International Conference on Control Applications, St.Petersburg, Russia, 2009.

[4] M. Bengtsson, Standardization Issues in Condition Based Maintenance, Proceeding of the 16th International Congress of Condition Monitoring and Diagnostic Engineering Management, Vaxjo, Sweden, 2003.

[5] G. Waeyenbergh, L. Pintelon, Maintenance concept development: A case study, International Journal of Production Economics, 89(2004) 395-405.

[6] K. Wang, W. Song, J. Li, The Use of an Autoregressive Integrated Moving Average Model for Prediction of the Incidence of Dysentery in Jiangsu, China, Asia Pacific Journal of Public Health, 28(2016) 336-346.

[7] R.K. Avuglah, K.A. Adupoku, E. Harris, Application of ARIMA models to road traffic accident cases in Ghana, International Journal of Statistics \& Applications, 4(2014) 233-239.

[8] E Harris, Modeling Annual Coffee Production in Ghana Using ARIMA Time Series Model, International Journal of Business \& Social Research , 2(2012) 175-186. 